\title{
Monopole Giant Resonances and TDHF boundary conditions
}

\author{
P. D. Stevenson ${ }^{\mathrm{a}}$, D. Almehed ${ }^{\mathrm{a}}$, P.-G. Reinhard ${ }^{\mathrm{b}}$, J. A. Maruhn ${ }^{\mathrm{c}}$ \\ aDepartment of Physics, University of Surrey, Guildford, GU2 7HX, United Kingdom \\ bInstitut für Theoretische Physik II, Universität Erlangen-Nürnberg, Staudtstrasse 7, \\ D-91058 Erlangen, Germany \\ 'Institut für Theoretische Physik, Universität Frankfurt, Robert-Mayer-Strasse 8-10, \\ D-60325 Frankfurt am Main, Germany
}

Using time-dependent Hartree-Fock, we induce isoscalar and isovector monopole vibrations and follow the subsequent vibrations of both the same and opposite isospin nature in the $N \neq Z$ nucleus ${ }^{132} \mathrm{Sn}$. By suitable scaling of the proton and neutron parts of the excitation operators, the coupling between the modes is studied, and the approximate normal modes found. Chaotic dynamics are then analysed in the isoscalar giant monopole resonance by using reflecting boundaries in a large space to build up a large number of $0^{+}$states whose spacings are then analysed. A Wigner-like distribution is found.

\section{Introduction}

In the time-dependent Hartree-Fock (THDF) approximation, nuclear dynamics can be studied with only the need to input an effective interaction and boundary and initial conditions. The small amplitude limit of TDHF is equivalent to RPA and thus forms a useful basis for the study of giant resonances. While TDHF is generally more complicated that RPA for simple cases, it scales well to e.g. triaxial systems [1] and naturally allows the study of nonlinearity [2]. It is also always fully self-consistent, whose importance has been recently emphasised [3].

\section{Formalism and Isospin Mixing}

In TDHF the nuclear wavefunction is represented at each point in time by a single Slater Determinant, whose initial single-particle states are given by a solution of the static Hartree-Fock equations, and which evolve in time according to

$i \hbar \dot{\phi}_{i}=\left\{\hat{h}[\rho]+\hat{f}_{\text {ext }}\right\} \phi_{i}$.

Here, $\hat{h}$ is the Hartree-Fock Hamiltonian, which itself depends on time owing to its dependence on the time-varying wavefunctions through the density. For an effective interaction, we use the Skyrme energy density functional [4], with all terms included in both the static and dynamic parts of the calculation. The equations are solved by first finding a static 

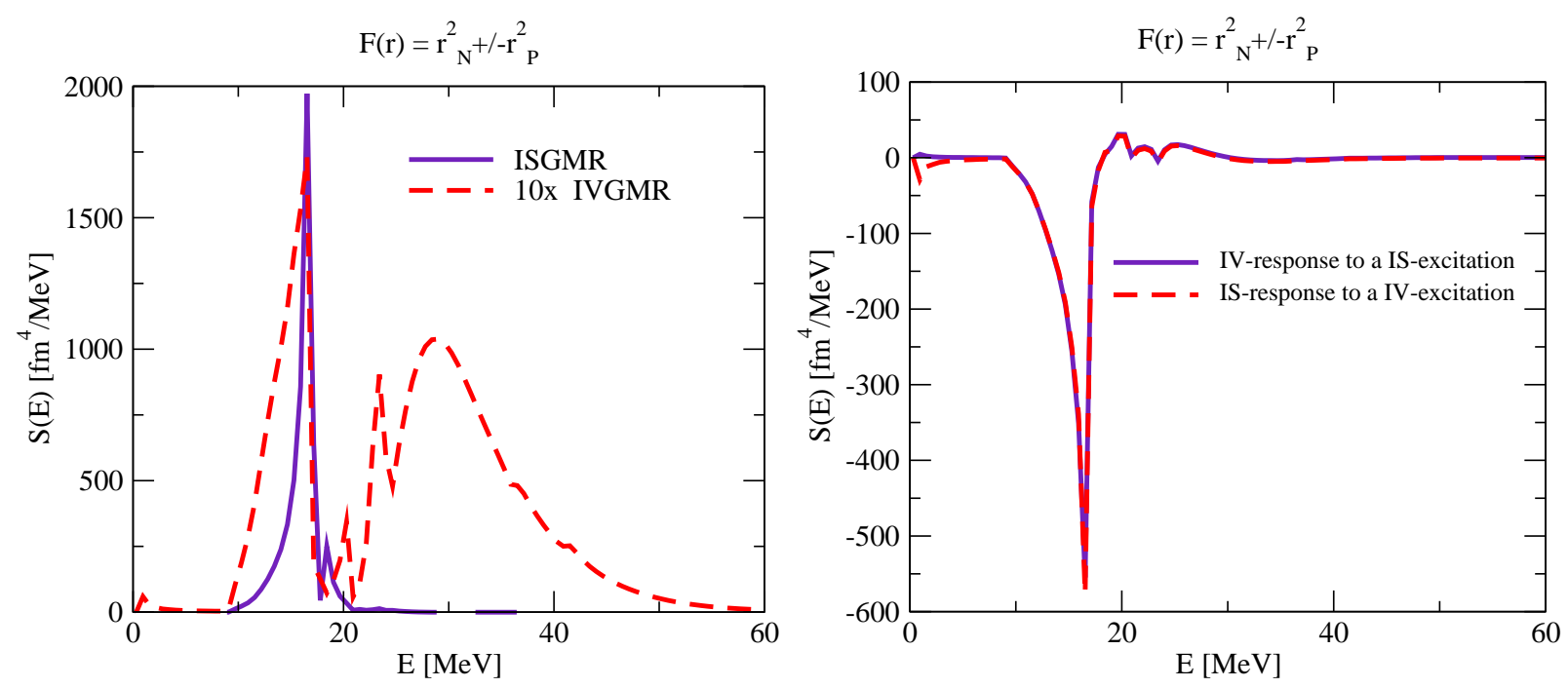

Figure 1. Direct (left) and cross-reponses (right) using simple difference between proton and neutron mean-square radii.

solution of the HF equations to give the $t=0$ wavefunctions, then allowing these wavefunctions to evolve according to

$\phi_{i}(t+\Delta t)=\sum_{k} e^{i\left(\hat{h}+\hat{f}_{e x t}\right) \Delta t} \phi_{i}(t)$

In (1) and (2) $\hat{f}_{\text {ext }}$ is an external field, which can be switched on to induce giant resonances. For example, one can consider an external field of the form

$f_{1}=\sum_{i} r_{i}^{2}$

to excite the isoscalar giant monopole resonance. One then observes the expectation value of this same operator as a function of time. The fourier transform of this time series then yields the strength function

$S(E)=\sum_{\nu}\left|\left\langle\nu\left|f_{1}\right| 0\right\rangle\right|^{2} \delta\left(E-E_{\nu}\right)$

though one may also follow the response of other operators to look at the mixing between modes [5]. For example, if one defined an isovector monopole operator as

$f_{2}=\sum_{i} \tau_{i}^{z} r_{i}^{2}$

then inducing $f_{1}$ at the beginning of the TDHF calculation but following $f_{2}$ as a function of time gives access to a mixed strength function

$S(E)=\sum_{\nu}\left\langle 0\left|f_{1}\right| \nu\right\rangle\left\langle\nu\left|f_{2}\right| 0\right\rangle \delta\left(E-E_{\nu}\right)$. 

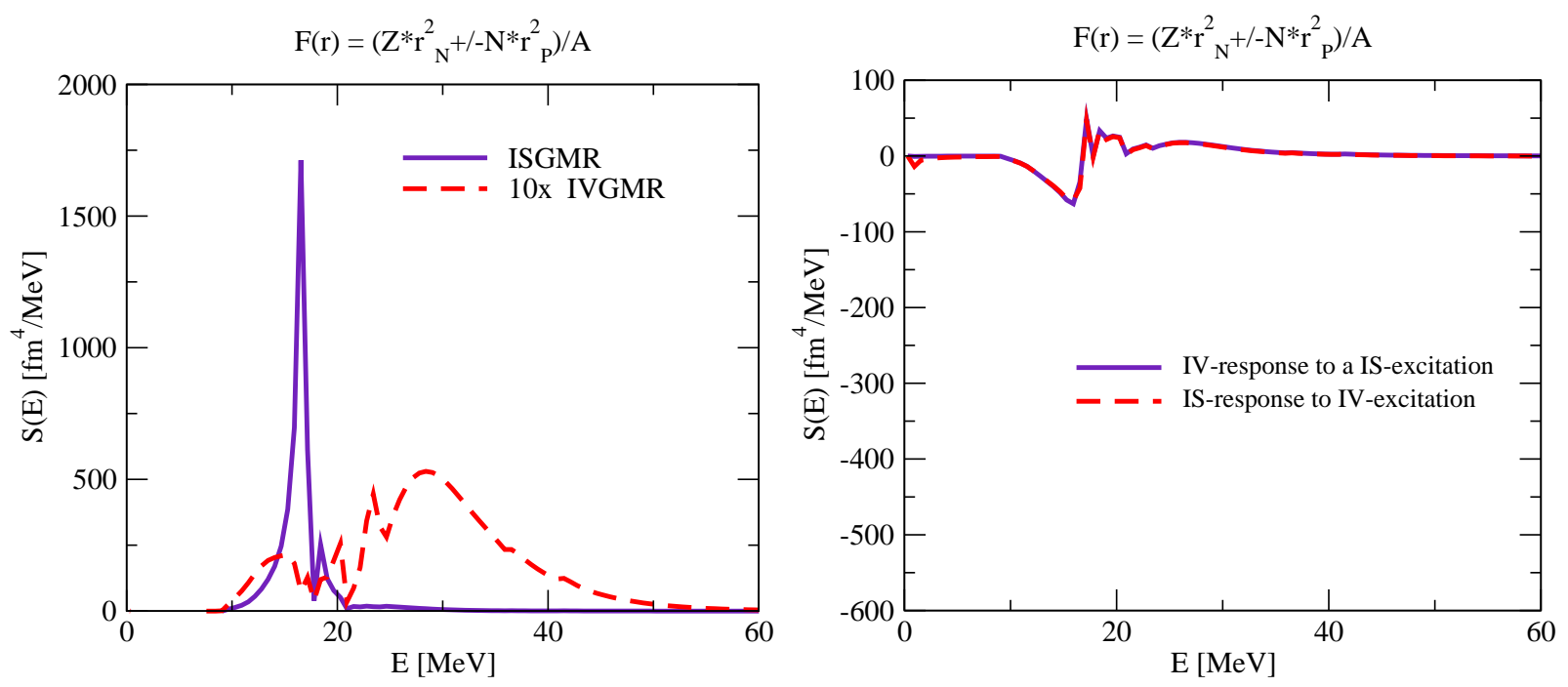

Figure 2. Direct (left) and cross-reponses (right) using weighted difference between proton and neutron mean-square radii.

Usually, the excitation functions (3) and (5) are called the isoscalar and isovector giant monopole excitations and are used to study the resonances of those names. However, if $N \neq Z$ these vibrations are not the eigenmodes of the system, and are strongly coupled together. It is interesting question to find the natural resonance modes which are of a mixed isospin nature. In the case of the dipole mode, it is clear that to decouple the spurious centre of mass motion associated with the leading order of the isoscalar dipole excitation when applying an isovector perturbation, one must relatively weight the proton and neutron parts of the excitation operator according to

$F(r)=\frac{Z}{A} \sum_{n} z_{n}-\frac{N}{A} \sum_{p} z_{p}$

Figures 1 and 2 show the effect of applying this same scaling to the monopole resonances in ${ }^{132} \mathrm{Sn}$ using the Skyrme force SkO. In figure 1, the usual situation applies, in which the ISGMR and IVGMR resonances are excited and observed with the usual $r_{n}^{2} \pm r_{p}^{2}$ operator, whereas in Figure 2 they are scaled in the manner of (7). In the unscaled case, one sees that the IVGMR response to the IVGMR excitation includes a substantial amount of strength in the region of the isoscalar peak, whereas in the scaled case this is substantially reduced.

The "mixed" strength functions, on the right of these two plots show that (i) the IV response to the IS kick is the same as the IS reponse to the IV kick, as it should be and confirming the numerical accuracy, and (ii) the coupling between the modes is greatly reduced when the scaled operators are used, but it is not eliminated. In fact, the mixing of the modes can differ as a function of energy, and it might not prove possible to find the normal modes of oscillation with so simple an Ansatz as the kind of scaling given in (7). Further investigations on this topic are underway. 


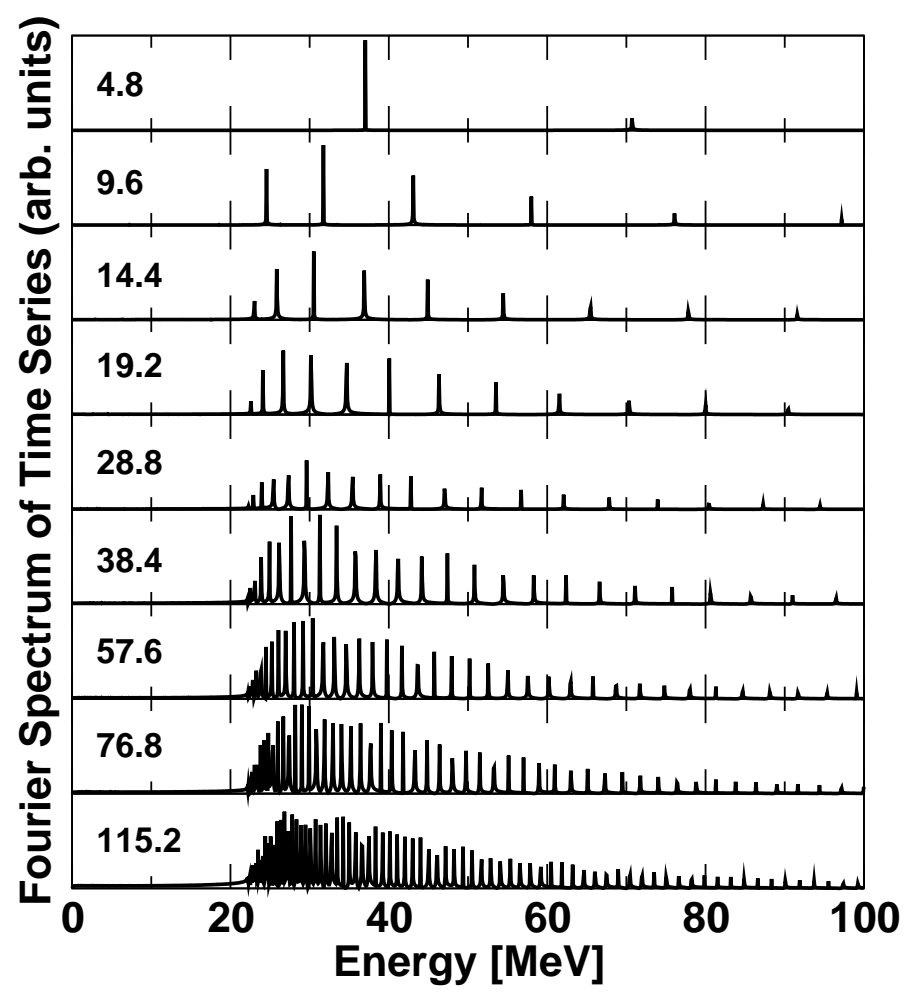

Figure 3. As the size of the space grows (given in fm by the numbers in the panes) the number of RPA eignenmodes in the Fourier power spectrum increases.

\section{Consequences of Boundary Condition}

As a second example of our work on giant monopole resonances with TDHF, we present some consequences of dealing with boundary conditions.

Our TDHF calculations are performed on a coordinate space grid which, in the special case of spherical symmetry appropriate for monopole vibrations in spherical nuclei, can be made arbitrarily large. Since giant resonances occur above the particle emission threshold, particle flux travels out from the nucleus towards the edge of the box as the TDHF calculation proceeds. By default, this flux will reflect from the edge of the box, and return to the nucleus, then drive its vibration. This unphysical effect turns out to reduce in a small amplitude TDHF calculation into a normal RPA as opposed to a continuum RPA calculation, and as such, is still a reasonable representation of the resonance. The strength functions are fragmented into a series of sharp peaks, but these nevertheless lie in the correct place and can be compared exactly with continuum calculations (available in TDHF with absorbing boundaries [6], [7]) if both are smoothed equally [8].

By using reflecting boundaries one has a system governed by a nonlinear driven differential equation, which is a good candidate for producingx chaotic motion, using the tools of classical chaos analysis. This kind of study has already been performed in the case of giant monopole resonances using the RMF framework [9], [10], and we do not wish to repeat the same analysis here. We wish, however, to look at the same issue from a 

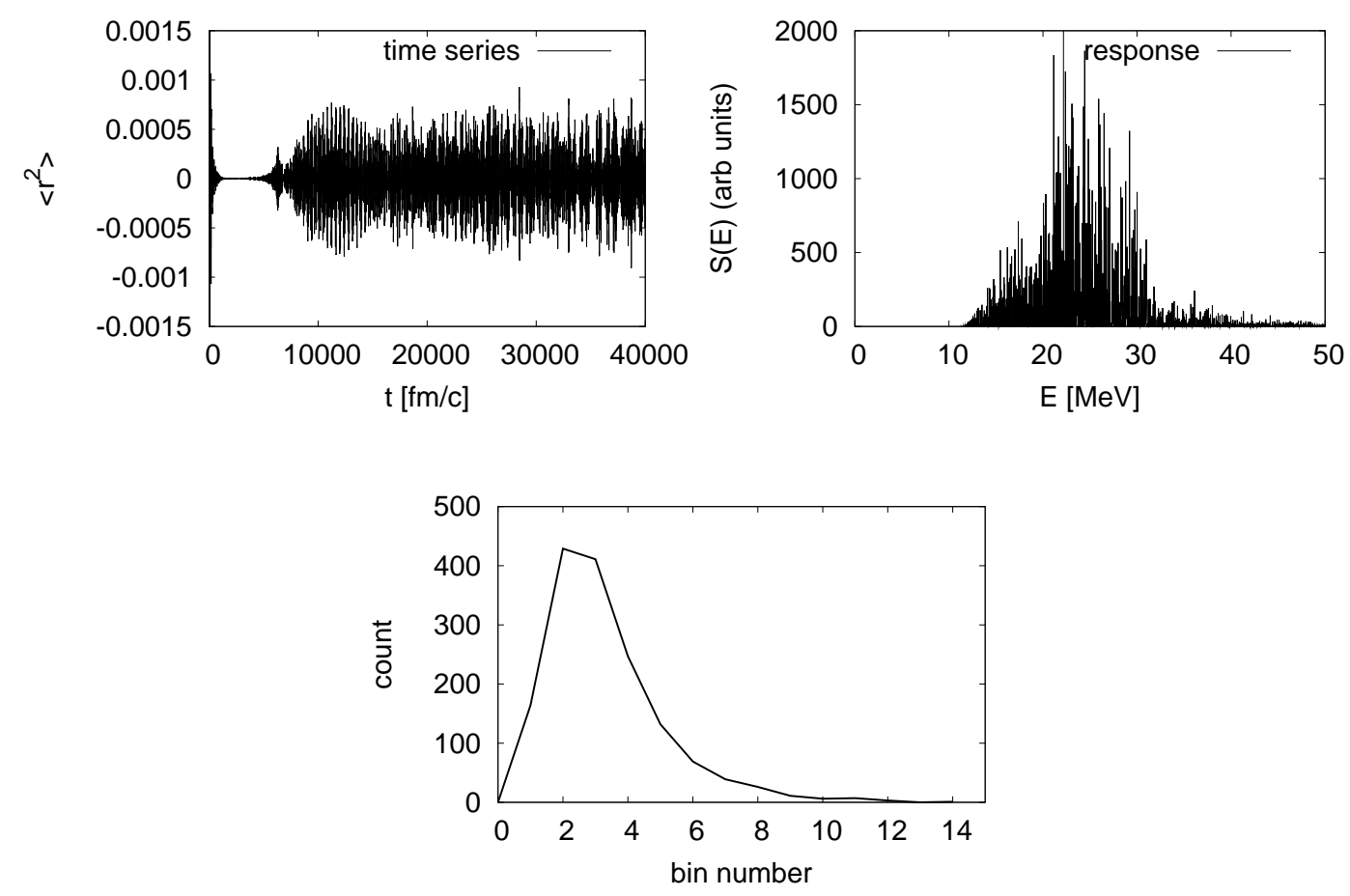

Figure 4. Time series of isoscalar repsonse to isoscalar excitation in ${ }^{16} \mathrm{O}$ (top left), strength function, showing discretised peaks (top right), and nearest neighbour distribution of peaks (bottom)

slightly different angle, and see what effect the size of the box in which one performs the TDHF calculation is. In the limit of taking the size of the box to be as small as possible, one is effectively reducing the number of RPA eignemodes that can be constructed in the phase space to the extent the only a single (harmonic) mode can be excited and a purely harmonic, non-chaotic, motion can be found. In the other limit of taking the box size to be very large, the motion will be complicated and made from an large number of closely spaced (in energy) RPA modes. As the box size is made larger, the structure of the strength function more and more approaches the continuum structure [7], as shown in the power spectra of Figure 3

By performing a calculation of an isoscalar giant monopole resonance in ${ }^{16} \mathrm{O}$, with the $\mathrm{SkM}^{*}$ Skyrme force in a large $(600 \mathrm{fm})$ box, for a long time $\left(2^{20} \mathrm{fm} / \mathrm{c}\right)$ to allow discretisation of the spectrum, a response was obtained which had a large number of $0^{+}$peaks. We then analysed the nearest neighbour spacing to see whether they followed Poisson or Wignerlike distributions - the key signature of quantum chaos.

Figure 4 shows the results of this calculation, including the first part of the time response of the isoscalar monopole operator (NB since this is an $\mathrm{N}=\mathrm{Z}$ nucleus the issues of the first part of this paper are not relevant), the corresponding strength function, consisting of a large number of sharp levels, and a histogram showing the nearest-neighbour distribution. 
The histogram shows the characteristic peak at finite separation, though does not quite fit a Wigner distribution. Despite the large box size used in the calculation, it is still finite, providing an artificial lower limit to the spacing, and further study of this effect should be made.

In conclusion, we have demonstrated calculations of giant resonances using TDHF with a full Skyrme force, and investigated the mixing between isoscalar and isovector motion, finding that by varying the weighting of the proton and neutron operators, the normal modes can be approximately reached. By performing what is essentially RPA in a very large space, the motion from the TDHF calculations, which has previously been subjected to the techniques of classical chaos analysis has been studied from the point of view of nearest-neighbour spacing. A possibly Wigner-like distribution was found.

\section{REFERENCES}

1. J. A. Maruhn, P.-G. Reinhard, P. D. Stevenson, J. R. Stone and M. R. Strayer, Phys. Rev. C 71, 064238 (2005)

2. C. Simenel and Ph. Chomaz, Phys. Rev. C 68, 024302 (2003)

3. Tapas Sil, S. Shlomo, B. K. Agrawal, P.-G. Reinhard, Phys. Rev. C 73, 034316 (2006)

4. Michael Bender, Paul-Henri Heenen and P.-G. Reinhard, Rev. Mod. Phys. 75, 121 (2003)

5. D. Almehed and P. D. Stevenson, J. Phys. G 31, S1819 (2005)

6. Takashi Nakatsukasa and Kazuhiro Yabana, Phys. Rev. C 71, 024301 (2005)

7. P.-G. Reinhard, P. D. Stevenson, D. Almehed, J. A. Maruhn and M. R. Strayer, Phys. Rev. E 73, 036709 (2006)

8. P. D. Stevenson, M. R. Strayer, J. Rikovska Stone and W. G. Newton, Int. J. Mod. Phys. E 13, 181 (2004)

9. G. A. Lalazissis, D. Vretenar, N. Paar and P. Ring, Chaos Solitons and Fractals 17, $585(2003)$

10. D. Vretenar, N. Paar, P. Ring, and G. A. Lalazissis, Phys. Rev. E 60, 308 (1997) 